\title{
Tunneling Gap as Evidence for Time-Reversal Symmetry Breaking at Surfaces of High-Temperature Superconductors
}

\author{
R. B. Laughlint \\ Department of Physics, Stanford University, Stanford, CA 94305 \\ and \\ Lawrence Livermore National Laboratory, P. O. Box 808, Livermore, CA 94550
}

\begin{abstract}
It is argued that recent Josephson junction and point-contact tunneling experiments, interpreted as intended by their authors, indicate that time-reversal symmetry breaking occurs at surfaces of cuprate superconductors. The variation among experiments and the failure of previous searches to find $T$-violation are ascribed to disorder and effects of 3-dimensionality. The "anyon" approach to the $t-J$ model is shown to predict a conventional BCS order parameter of $d_{x^{2}-y^{2}}+i \epsilon d_{x y}$ symmetry, with $\epsilon$ roughly 3 times the doping fraction $\delta$, which is consistent with these experiments but not demonstrated by them.
\end{abstract}

74.20.Kk, 74.20.Mn, 74.50.+r

Typeset using REVTEX 
The purpose of this Letter is to point out that time-reversal symmetry breaking [1], the key prediction of the "anyon" approach to the high-temperature superconductivity problem, may already have been demonstrated in a series of recent experiments conducted for other purposes. While it is wise to be cautious, particularly in light of previous failures to detect $T$-violation [2] and the possibility that one or more of these experiments may later prove to be wrong or misinterpreted, the implication of the experiments as they now stand is clear. The relevant experiments are the photoemission [3] and light scattering [四] measurements of the gap anisotropy, the microwave measurements of the low-temperature conductivity [5], the Josephson phase coherence experiments of Wollman et al. [6], and the scanning tunneling microscope measurements of Hasegawa et al. [0] reporting an intrinsic energy gap. The essence of the argument is that all of these except the last point to the occurrence of a conventional BCS order parameter of $d_{x^{2}-y^{2}}$ symmetry, the behavior commonly found in theories based on incipient antiferromagnetism of the conducting electrons [8]. This, however, is fundamentally incompatible with the last experiment unless the order parameter is complex, which is impossible unless the ground state violates $T$. Thus the simultaneous occurrence of $d$-wave superconductivity and an energy gap in any part of the sample, if true, constitutes definitive evidence for $T$-violation.

A $d_{x^{2}-y^{2}}$ order parameter $\Delta_{k}$ is distinguished from a conventional $s$-wave order parameter by sign reversals. In high-Tc superconductors this is most easily discussed in terms of the idealized electron energy band

$$
E_{k}^{0}=-2 t_{0}\left[\cos \left(k_{x} b\right)+\cos \left(k_{y} b\right)\right]
$$

where $t_{0}$ is an energy parameter and $b$ is the bond length of a 2-dimensional square lattice. One imagines creating a fermi sea by filling the states with $E_{k}<0$. Allowing the electrons to interact through weak near-neighbor spin exchange [8] then leads to a superconducting state for which the quasiparticles have energies

$$
E_{k}= \pm \sqrt{\left(E_{k}^{0}\right)^{2}+\left|\Delta_{k}\right|^{2}}
$$

where 


$$
\Delta_{k}=\Delta^{0}\left[\cos \left(k_{x} b\right)-\cos \left(k_{y} b\right)\right] .
$$

The gap parameter $\Delta_{k}$ is positive in the $x$-direction, negative in the $y$-direction, and zero at the nodes in between. In an $s$-wave superconductor $\Delta_{k}$ is the constant $\Delta^{0}$.

Of the experiments listed above, only the Josephson experiment of Wollman et al. [6] can directly sense the sign reversal of $\Delta_{k}$, even in principle. It accomplishes this by detecting electric currents spontaneously generated in a loop of $\mathrm{Pb}$ wire connected between the $x$ - and $y$-faces of a high-Tc superconductor. Although the result reported by Wollman et al. [6] is positive and consistent with the large body of circumstantial evidence for $d$-wave pairing, it has not yet been reproduced and is quite controversial. In what follows we shall assume that this experiment is right. It must be emphasized that it is the only direct evidence we have for sign reversal of $\Delta_{k}$.

This experiment is supported by considerable indirect evidence for $d$-wave superconductivity with nodes at $k=( \pm \pi / 2 b, \pm \pi / 2 b)$, in particular by the presence in all samples of low-energy excitations in the superconducting state. The evidence is, unfortunately, complicated by the disorder effects that plague these materials. For example, Giaver tunneling finds states in the gap so commonly that the claim of Hasegawa et. al. [7] to have observed a clean gap, the experiment motivating this Letter, is widely questioned. At the same time, the large zero-bias conductance seen in most tunneling experiments is commonly dismissed as an artifact of disorder at the tunnel contact [9]. The $T^{2}$ deviation of the penetration depth from its zero-temperature value, and its crossover to linear- $T$ behavior above about $5 K$, seen in high-quality samples [10] is consistent with $d_{x^{2}-y^{2}}$ superconductivity only if disorder that is difficult or impossible to eliminate from the samples [11] is assumed to exist. The same is true of the zero-temperature microwave conductivity [5.10]. The intrinsic heat capacity below $10 \mathrm{~K}$ is not known for any high- $T_{c}$ superconductor because the signal is always swamped by a large Schottkey-like heat capacity [12] similar to that expected of a spin glass. That at least some of these excitations are intrinsic and attributable to a $d$-wave node is indicated by several less accurate or model-dependent experiments, the most accessible of 
which is the angle-resolved photoemission work of Shen et al. [3]. This reports measureable changes to the quasiparticle energies $E_{k}$ resulting from cooling the sample through its superconducting transition except near this special value of $k$. The complex temperature and polarization dependence of inelastic light scattering is accounted for quantitatively by $d_{x^{2}-y^{2}}$ superconductivity if reasonable assumptions are made about the relevant matrix elements ${ }^{4}$. The same is true for the extensive magnetic resonance data [13]. While the situation is still confusing and controversial, it is clear that a large body of experimental evidence is consistent with the simultaneous presence in all samples of both disorder and a $d$-wave node.

Let us now consider the tunneling experiment of Hasegawa et al. [7]. It is very important for our argument that this experiment was performed with a scanning tunneling microscope tip, that it reported a well-developed energy gap only at certain places on the sample, and that it was inconsistent with conventional thin-film tunnel junction experiments, which never reveal a full gap [9]. Barring the possibility that it is an experimental artifact, such as coulomb blockade [14 or an effect of anisotropic tunneling, this result implies that a genuine energy gap in the quasiparticle spectrum develops at some places on the surface. The cleanliness of the reported gap suggests that this gap is intrinsic. Why it should develop only on islands is an open question. One possiblity is that it is destroyed almost everywhere on the surface by disorder. That disorder has the potential to do this, provided that the order parameter has sign reversals, is well known [11. This explanation probably cannot account for the absence of the gap in the bulk, although further experimental studies are required to tell for certain. A more likely explanation in this case is that the gap is suppressed because of the magnetic fields it would create. However, regardless of the mechanism by which the gap is destroyed, its existence anywhere in the sample indicates $T$-violation, since an energy gap is forbidden as a matter of principle in a $d$-wave superconductor with a real order parameter.

Let us now consider the specific complex order parameter 


$$
\Delta_{k}^{\text {chiral }}=\Delta^{0}\left[\cos \left(k_{x} b\right)-\cos \left(k_{y} b\right)+i \epsilon \sin \left(k_{x} b\right) \sin \left(k_{y} b\right)\right]
$$

where $\epsilon$ is a number, implicit in the anyon technique. Because the imaginary part of this order parameter has sign reversals, it is susceptible to destruction by non-magnetic disorder 111 and thus consistent with the idea that $\epsilon$ might be zero everywhere except on islands. That there should be such an order parameter was first suggested by Rokhsar [15], who also pointed out that it was potentially inconsistent with the known phase diagram of these materials. Since the real and imaginary parts of $\Delta_{k}^{c h i r a l}$ lie in different irreducible representations of the lattice point group, they cannot mix in the Ginzberg-Landau functional, and thus must develop as separate order parameters as the temperature is lowered. This would give 2 phase transitions, rather than the observed 1 . However, the suppression of the the smaller $d_{x y}$ order parameter almost everywhere in the sample would resolve this paradox by preventing the acquisition of long-range order and killing the lower phase transition. It would also kill all effects of global $T$-violation, and account for the failure of previous searches to find them [2].

Let us now show that this order parameter is implicit in the anyon approach to the $t-J$ Hamiltonian

$$
\mathcal{H}_{t-J}=\sum_{<j, k>}\left\{-t \sum_{\sigma} c_{j \sigma}^{\dagger} c_{k \sigma}+\frac{J}{2} S_{j} \cdot S_{k}\right\}
$$

and estimate its magnitude. It should be noted such an order parameter is not uniquely obtained this way, but also arises in certain simple BCS Hamiltonians [16. As usual, $S_{j}$ denotes the spin operator for the $j^{\text {th }}$ site, $\langle j, k>$ denotes a sum on near-neighbor pairs, with each pair counted twice, and $J=0.1 \mathrm{eV}$ and $t=0.5 \mathrm{eV}$ are the spin-exchange and electron hopping matrix elements. We assume that the lattice has $N$ sites and $M=N \delta$ holes.

The anyon superconducting state for the $t-J$ model is an electron wavefunction of the form

$$
|\Psi\rangle=\sum_{\ell_{1}, \ldots, \ell_{M}} a_{\ell_{1}, \ldots, \ell_{M}}\left|\ell_{1}, \ldots, \ell_{M}\right\rangle
$$


where $\left|\ell_{1}, \ldots, \ell_{M}\right\rangle$ is a basis wave function describing "holons" at sites $\ell_{1}, \ldots, \ell_{M}$. This is given explicitly by [17]

$$
\left|\ell_{1}, \ldots, \ell_{M}\right\rangle=\prod_{j}^{N}\left(1-n_{j \uparrow} n_{j \downarrow}\right) \prod_{\alpha}^{M}\left(1-n_{\ell_{\alpha} \uparrow}-n_{\ell_{\alpha} \downarrow}\right)\left|\Psi_{\text {flux }}\right\rangle
$$

where $n_{j \sigma}=c_{j}^{\dagger} \sigma^{c} j \sigma$ is the number operator for an electron of spin $\sigma$ at site $j$, and $\left|\Psi_{\text {flux }}\right\rangle$ is the ground state of the commensurate flux Hamiltonian

$$
\mathcal{H}_{\text {flux }}=-t \sum_{<j, k>} \sum_{\sigma} \exp \left\{i \frac{\pi}{2}(1-\delta)\left(x_{j}-x_{k}\right)\left(y_{j}+y_{k}\right) / b^{2}\right\} c_{j \sigma}^{\dagger} c_{k \sigma}
$$

with $N-M$ electrons. $\mathcal{H}_{\text {flux }}$ violates $T$, as is required for the holon basis to be defined [17]. It is also possible to construct the basis using $\mathcal{H}_{\text {flux }}^{*}$, in which case $T$ is violated in the opposite sense. The holons defined by Eq. (7) obey $1 / 2$ fractional statistics [17] in that varying the expansion coefficients in Eq. (6) to minimize the expected energy $\left\langle\Psi\left|\mathcal{H}_{t-J}\right| \Psi\right\rangle$ is equivalent, when $\delta$ is small, to solving the fermion problem [17]

$$
\begin{aligned}
\mathcal{H}_{\text {anyon }} & =\sum_{j} \frac{\hbar^{2}}{2 m^{*}}\left|P_{j}+\frac{e}{c} A_{j}\right|^{2} \\
A_{j} & =\frac{1}{2} \frac{\hbar c}{e} \sum_{k \neq j} \frac{\hat{z} \times\left(r_{j}-r_{k}\right)}{\left|r_{j}-r_{k}\right|^{2}}
\end{aligned}
$$

with isospin. The latter corresponds to the valley degeneracy of the holon band structure, which in the $\delta \rightarrow 0$ limit is described by Eq. (8) without spin [17]. Since the holon dispersion relation in this limit is given by

$$
E_{k}^{\text {holon }} \cong \pm 2 t \sqrt{\cos ^{2}\left(k_{x} b\right)+\cos ^{2}\left(k_{y} b\right)}
$$

we find that the valley minima occur at $k=(0,0)$ and $(\pi / b, 0)$ and are characterized by the mass $m^{*} \cong \hbar^{2} /\left(\sqrt{2} b^{2} t\right)$.

The first step in the order parameter calculation is to compute the non-local order parameter of the continuum anyon gas described by Eq. (9) [18. We will take the ground state to be

$$
\left|\Phi_{\text {anyon }}\right\rangle \cong \prod_{q}\left\{\frac{1}{2} \exp \left(-|q| / \sqrt{\pi \rho} \rho_{q} \rho_{-q}\right)\right\}\left|\Phi_{H F}\right\rangle
$$


where $\rho=\delta / b^{2}$ is the particle density, $\rho_{q}=\Sigma_{j} \exp \left(i q \cdot r_{j}\right)$ is the density operator, and $\left|\Phi_{H F}\right\rangle$ is the ground state of the Hamiltonian obtained by substituting the mean-field vector potential $\langle A\rangle=h c /(2 e) \rho y \hat{x}$ for $A_{j}$ in Eq. (9). The prefactor in this expression is the usual modification of the Hartree-Fock ground state implicit in the random phase approximation. Let $\psi_{+}(z)$ and $\psi_{-}(z)$ denote the operators annihilating holons with "up" and "down" isospin, respectively, at $z=x+i y$ in the fermi representation. The nonlocal order parameter is given in terms of these by 18

$$
\begin{aligned}
& \int\left\langle\Phi_{\text {anyon }}\left|\psi_{+}^{\dagger}\left(z_{1}\right) \psi_{-}^{\dagger}\left(z_{1}\right)\left\{\psi_{+}^{\dagger}(z) \psi_{+}(z)+\psi_{-}^{\dagger}(z) \psi_{-}(z)\right\} \psi_{+}\left(z_{2}\right) \psi_{-}\left(z_{2}\right)\right| \Phi_{\text {anyon }}\right\rangle \\
& \times \frac{\left(z_{1}^{*}-z^{*}\right)}{\left|z_{1}-z\right|} \frac{\left(z_{2}-z\right)}{\left|z_{2}-z\right|} d^{2} z \quad\left|z_{1}-z_{2}\right| \rightarrow \infty \quad(1.9 \rho)^{2}
\end{aligned}
$$

The numerical value of $1.9 \rho$ reported here for the first time is obtained using the hypernetted chain technique [17]. We will adopt the notation $\left\langle\psi_{+}(z) \psi_{-}(z)\right\rangle=1.9 \rho$ as shorthand for this result.

The second step is to convert this continuum order parameter to the site basis. The unitary transformation relating $\psi_{+}(z)$ and $\psi_{-}(z)$ to $\psi(j)$, the fermi operator to annihilate a holon at site $r_{j}=\left(\ell_{j}, m_{j}\right) b$, is simply the the matrix of 1-body eigenstates of Eq. (8) at the two valley minima. We thus have

$$
\psi(j) \cong \sqrt{2} b\left\{\cos \left[\left(\frac{1}{8}-\frac{1}{2} m_{j}^{2}\right) \pi\right] \psi_{+}(z)+(-1)^{\ell_{j}} \sin \left[\left(\frac{1}{8}+\frac{1}{2} m_{j}^{2}\right) \pi\right] \psi_{-}(z)\right\}
$$

In obtaining this expression, we have imagined the sample to be divided into 4-site cells, and that $z$ defines the center of the cell containing $j$. Then, substituting Eq. (13) into Eq. (12), and using the fact that $\psi_{+}(z)$ and $\psi_{-}(z)$ anticommute with themselves and each other, we obtain

$$
\begin{aligned}
& \langle 0|\Psi(1) \Psi(2)| 0\rangle \cong 1.9 \delta(-1)^{\ell_{1}+m_{2}+\left(\ell_{1}+\ell_{2}\right) m_{1}} \\
& \times \frac{1}{\sqrt{2}}\left(\left[1-(-1)^{\ell_{1}+\ell_{2}+m_{1}+m_{2}}\right]-\left[1-(-1)^{\left(\ell_{1}+\ell_{2}\right)\left(m_{1}+m_{2}\right)}\right]\right)
\end{aligned}
$$

The third step is to evaluate the matrix element of $c_{j \uparrow}^{\dagger} c_{k \downarrow}^{\dagger}$ to annihilate a holon pair into the vacuum. This matter has been studied extensively in previous papers [17] and is too involved to discuss in detail here. We shall simply quote the result 


$$
\begin{aligned}
& \left\langle\ell_{1}^{\prime}, \ldots, \ell_{M}^{\prime}\left|c_{k^{\prime} \downarrow} c_{j^{\prime} \uparrow} c_{j \uparrow}^{\dagger} c_{k \downarrow}^{\dagger}\right| \ell_{1}, \ldots, \ell_{M}\right\rangle \cong \\
& \delta_{\ell_{1} j} \delta_{\ell_{2} k} \delta_{\ell_{1}^{\prime} j^{\prime}} \delta_{\ell_{2}^{\prime} k^{\prime}} \prod_{\alpha=3}^{M} \delta_{\ell_{\alpha} \ell_{\alpha}^{\prime}} \frac{\left(z_{j}^{\prime *}+z_{k}^{\prime *}\right) / 2-z_{\alpha}^{*}}{\left|\left(z_{j}^{\prime}+z_{k}^{\prime}\right) / 2-z_{\alpha}\right|} \frac{\left(z_{j}+z_{k}\right) / 2-z_{\alpha}}{\left|\left(z_{j}+z_{k}\right) / 2-z_{\alpha}\right|} \\
& \times\left\langle j^{\prime}, k^{\prime}\left|c_{j^{\prime} \uparrow} c_{k^{\prime} \downarrow}\right| 0\right\rangle\left\langle 0\left|c_{j \uparrow}^{\dagger} c_{k \downarrow}^{\dagger}\right| j, k\right\rangle+\text { cyclic permutations }
\end{aligned}
$$

where $|0\rangle$ denotes the state with no holons and $z_{j}=\left(\ell_{j}+i m_{j}\right) b$. The product on $\alpha$ in this expression is the same "unwinding" factor appearing in Eq. (12) and is the microscopic justification for its inclusion in Eq. (12). The remaining factor is given approximately by

$$
\begin{aligned}
\left\langle 0\left|c_{1 \uparrow}^{\dagger} c_{2 \downarrow}^{\dagger}\right| j, k\right\rangle \cong & \frac{\left(z_{1}-z_{2}\right)}{\left|z_{1}-z_{2}\right|} i^{\left(\ell_{1}-\ell_{2}\right)\left(m_{1}+m_{2}\right)}(-1)^{\ell_{1}+m_{1}} \\
& \times\left[\begin{array}{cl}
(1-\delta) \exp \{-\pi / 4(1-\delta)\} & ; 1 \text { and } 2 \text { near neighbors } \\
\delta \exp \{-\pi \delta\} & ; 1 \text { and } 2 \text { second neighbors } \\
0 & ; \text { otherwise }
\end{array}\right]
\end{aligned}
$$

The final step is to combine Eq. (15) and (16) with Eq. (14) to obtain an expression for $\left\langle c_{j \uparrow}^{\dagger} c_{k \downarrow}^{\dagger}\right\rangle$. This is accomplished by multiplying together the right sides of Eqs. (14) and (16) and then dividing out the factor $\left(z_{1}-z_{2}\right) /\left|z_{1}-z_{2}\right|$. This latter step accounts for the transformation of the basis functions defined by Eq. (7), which are symmetric under interchange of the holon positions, to their fermi representation. We obtain finally

$$
\begin{aligned}
\left\langle c_{1 \uparrow}^{\dagger} c_{2 \downarrow}^{\dagger}\right\rangle \cong i^{\left(\ell_{1}-\ell_{2}\right)\left(m_{2}-m_{1}\right)}(-1)^{m_{1}-m_{2}} & \\
& \times 1.9 \delta\left[\begin{array}{cl}
\sqrt{2}(1-\delta) \exp \{-\pi / 4(1-\delta)\} & ; 1 \text { and } 2 \text { near neighbors } \\
-2 \delta \exp \{-\pi \delta\} & ; 1 \text { and } 2 \text { second neighbors } \\
0 & ; \text { otherwise }
\end{array}\right]
\end{aligned}
$$

This is equivalent to Eq. (4) with

$$
\epsilon=\frac{\sqrt{8} \delta}{1-\delta} e^{(1-5 \delta) \pi / 4}
$$

Let us now make some comments about this result. The first is that it is quite crude and should be compared with experiment carefully. For example, it makes the unphysical prediction that superconductivity occurs in the $t-J$ model at any value of $J, t$, and $\delta$. A more 
formal development of the anyon approach using gauge theory techniques [19] cures problems of this kind, but is too technical to discuss here. Let us simply state the main results. i.) For realistic values of $J / t$ the order parameter is significantly smaller than Eq. (17) predicts, due to retardation effects. ii.) The equations have an antiferromagentic instability for $\delta \leq .05$ that depends weakly on $J / t$. iii.) The commensurate flux band structure of Eq. (6) has a gap collapse at $\delta \cong 1 / 3$ which causes calculations for doping fractions larger than this to be unreliable. That the calculation finds conventional superconducting order and $d$-wave symmetry is not surprising. The anyon approach is a legitimate variational technique for the $t$ - $J$ model, which is known by more reliable methods to have a tendency to $d$-wave pairing [20]. Also, it has been known for several years that "flux" vacua are fundamentally related to $d$-wave superconducting states [21]. The calculation is significant mainly because it predicts that superconducting pairing by means of "spin fluctuations" tends naturally to an order parameter with $d_{x^{2}-y^{2}}+i \epsilon d_{x y}$ symmetry, with $\epsilon$ significantly large.

I wish to thank M. R. Beasley, A. Kapitulnik, T. H. Geballe, S. Doniach, Z. Zou, A. A. Abrikosov, and D. J. Scalapino for numerous helpful discussions. The work was supported primarily by the National Science Foundation under Grant No. DMR-88-16217. Additional support was provided by the NSF MRL Program through the Center for Materials Research at Stanford University. 


\section{REFERENCES}

* e-mail: rbl@large.stanford.edu

[1] X.-G. Wen, F. Wilczek, and A. Zee, Phys. Rev. B39, 11413 (1989).

[2] S. Spielman et al, Phys. Rev. Lett. 69, 123 (1990); T.W. Lawrence, A Szöke, and R. B. Laughlin, Phys. Rev. Lett. 69, 439 (1992).

[3] Z.-X. Shen et al, Phys. Rev. Lett. 70, 1553 (1993); ibid., J. Phys. Chem. Solids 53, 1583 (1992); D. S. Dessau et al, ibid. 52, 1401 (1991); B. O. Wells et al, Phys. Rev. B46, 11830 (1992).

[4] T. P. Devereaux et al, Phys. Rev. Lett. 72, 396 (1994); T. Staufer, et al, Phys. Rev. Lett. 68, 1069 (1992).

[5] D. A. Bonn, et al, Phys. Rev. B47, 11314 (1993).

[6] D. A. Wollman et al, Phys. Rev. Lett. 72, 2134 (1993).

[7] T. Hasegawa et al, J. Phys. Chem Solids 54, 1351 (1993).

[8] S. R. White et al., Phys. Rev. B39, 839 (1989); P. Monthoux and D. Pines, Phys. Rev. Lett. 69, 961 (1992).

[9] J. M. Valles, Jr. et al., Phys. Rev. B44, 11986 (1991); D. Mandrus et al, Nature 351, 460 (1991).

[10] Z. Ma et al., Phys. Rev. Lett. 71, 781 (1993); W. Hardy et al., Phys. Rev. Lett. 70, 3999 (1993); S. Anlage et al. Phys. Rev. B44, 9764 (1991).

[11] M. Prohammer and J.P. Carbotte, Phys. Rev. B43, 5370 (1991); J. Annett, N. Goldenfeld, and S. R. Renn, Phys. Rev. B43, 2778 (1991); F. Gross et al. Z. Phys. B64, 175 (1986); R. J. Radtke, K. Levin, H.-B. Schüttler, and M. R. Norman, Phys. Rev. B48, 653 (1993); P. J. Hirschfeld, W. O. Putikka, and D. J. Scalapino, Phys. Rev. Lett. 71, 3705 (1993). 
[12] N. E. Phillips, R. A. Fisher, and J. E. Gordon, Prog. Low Temp. Phys. 13, 267 (1992).

[13] N. Bulut and D. J. Scalapino, Phys. Rev. Lett. 68, 706 (1992); J. A. Martindale et al., ibid. 68, 702 (1992).

[14] P. A. Lee, Phys. Rev. Lett. 71, 1887 (1993).

[15] D. S. Rokhsar, Phys. Rev. Lett. 70, 493 (1993).

[16] F. Wenger and S. Östlund, Phys. Rev. B47, 5977 (1993); F. Wenger, Licentiate Thesis, U. of Gothenburg, 1993).

[17] Z. Zou, J. L. Levy, and R. B. Laughlin, Phys. Rev. B45, 993 (1992); Z. Zou and R. B. Laughlin, Phys. Rev. B42, 4073 (1990); R. B. Laughlin and Z. Zou, Phys. Rev. B41, 664 (1989) ; P. Béran and R. B. Laughlin, Phys. Rev. B48, 10382 (1993).

[18] S. M. Girvin et al., Phys. Rev. Lett. 65, 1671 (1990).

[19] A. M. Tikofsky, R. B. Laughlin, and Z. Zou, Phys. Rev. Lett. 69, 3670 (1992).

[20] E. Dagotto and J. Riera, Phys. Rev. Lett. 70, 682 (1993).

[21] F. C. Zhang, C. Gros, T. M. Rice, and H. Shiba, Supercond. Sci. Technol. 1, 36 (1988). 